\section{Aqueous humor endothelin-1 and total retinal blood flow in patients with non- proliferative diabetic retinopathy}

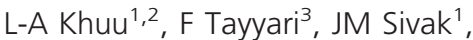

JG Flanagan ${ }^{1,2,3}$, S Singer ${ }^{2}$, MH Brent ${ }^{2}$,

D Huang ${ }^{4}, \mathrm{O} \mathrm{Tan}^{4}$ and C Hudson ${ }^{1,2,3}$

\begin{abstract}
Purpose The purpose of this study was to determine the association between aqueous ET-1 levels and total retinal blood flow (TRBF) in patients with non-insulindependent type 2 diabetes mellitus (T2DM) and early non-proliferative diabetic retinopathy (NPDR).

Patients and methods A total of 15 agematched controls and 15 T2DM patients with NPDR were recruited into the study. Aqueous humor $(\sim 80-120 \mu 1)$ was collected before cataract surgery to measure the levels of ET-1 using suspension multiplex array technology. Four weeks post surgery, six images were acquired to assess TRBF using the prototype RTVue Doppler FD-OCT (Optovue, Inc., Fremont, CA, USA) with a double circular scan protocol. At the same visit, forearm blood was collected to determine plasma glycosylated hemoglobin (A1c) levels.
\end{abstract}

Results Aqueous ET-1 was significantly elevated in the NPDR group compared with the control group $(3.5 \pm 1.8$ vs $2.2 \pm 0.8$, $P=0.02)$. TRBF was found to be significantly reduced in the NPDR group compared with the control group $(34.5 \pm 9.1$ vs $44.1 \pm 4.6 \mu \mathrm{l} / \mathrm{min}, P=0.002)$. TRBF and aqueous ET-1 were not correlated within the NPDR group $(r=-0.24, P=0.22)$. In a multivariate analysis, high A1c was associated with reduced TRBF and aqueous ET-1 levels across control and NPDR groups $(P<0.01)$. Conclusion Aqueous ET-1 levels were increased while TRBF was reduced in patients with NPDR compared with the control group. Although not directly associated, the vasoconstrictory effects of ET-1 are consistent with a reduced TRBF observed in early DR. ET-1 dysregulation may contribute to a reduction in retinal blood flow during early DR.

Eye (2017) 31, 1443-1450; doi:10.1038/eye.2017.74; published online 26 May 2017

\section{Introduction}

Diabetic retinopathy (DR) is a microvascular complication of the retina and is currently the leading cause of blindness in working age population with diabetes mellitus. Following 20 years of diabetes, a majority of patients with type 1 and type 2 diabetes mellitus will suffer from some form of retinopathy. ${ }^{1,2}$ Alterations in retinal blood flow are one of the earliest abnormalities observed in diabetes. Decreased blood flow has been measured in both animals ${ }^{3,4}$ and humans. ${ }^{5-8}$ Although impairments in retinal blood flow in early diabetes have been reported, early pathogenic mechanisms in early DR are unknown.

Endothelin-1 (ET-1), an endothelial-derived vasoactive factor, is an important regulator of vessel tone and blood flow. ${ }^{9,10}$ ET-1 is derived from 'big endothelin', an inactive pre-propeptide that is cleaved by endothelin-converting enzyme. ${ }^{11}$ In the eye, ET-1 is synthesized by the vascular endothelium, ${ }^{11}$ retinal pigmented epithelium, ${ }^{12}$ and non-pigmented ciliary epithelium. ${ }^{13}$ The potency and wide distribution of ET-1 in the anterior and posterior poles of the eye suggest an important physiological role in the regulation of the human ocular circulation, especially in diabetes. ${ }^{14,15}$ ET-1 regulates ocular perfusion primarily by modulating vascular smooth muscle $3,10,16$ and pericytes contractility. ${ }^{17,18}$ Intravitreal administration of exogenous ET-1 reduces retinal and optic nerve head blood flow, ${ }^{19}$ leading to localized ischemic retinal damage. ${ }^{20}$ Furthermore, several ocular
${ }^{1}$ Institute of Medical Science, University of Toronto, Toronto, ON, Canada

${ }^{2}$ Vision Science and Ophthalmology, Toronto Western Hospital, Toronto, ON, Canada

${ }^{3}$ School of Optometry and Vision Science, University of Waterloo, Waterloo, ON, Canada

${ }^{4}$ Casey Eye Institute, Oregon Health and Science University, Portland, OR, USA

Correspondence: C Hudson, School of Optometry and Vision Science, University of Waterloo, 200 University Ave. West, Waterloo, ON

N2L 3G1, Canada

Tel: +1 5198884567 ext 35562;

Fax: +1 5197250784

E-mail: chudson@

uwaterloo.ca

Received: 19 July 2015 Accepted in revised form: 14 March 2017 Published online: 26 May 2017 
diseases have been associated with the activation of the ET system, including retinal vascular occlusion ${ }^{21-23}$ and open-angle glaucoma. ${ }^{24-26}$

Accumulating studies have proposed that early dysregulation of ET-1 could be the principal cause of the altered retinal blood flow in the early stages of DR. In early experiments by Takagi et al, ${ }^{4}$ activation of the endogenous ET system in diabetic rats appeared to be associated with reductions in retinal blood flow, and subsequent treatment using BQ-123, an $\mathrm{ET}_{\mathrm{A}}$ antagonist, normalized blood flow in this model. Additionally, evidence from streptozotocin-induced diabetic rat model showed an increase in ET peptide and ET receptors in the diabetic retina, ${ }^{27}$ which impairs retinal pericyte activity, ${ }^{28}$ and influences capillary blood flow. Although endogenous ET-1 levels in the vitreous $^{29-32}$ and plasma ${ }^{33,34}$ are elevated during DR, the functional significance of ocular ET-1 levels to early retinal blood flow hemodynamics have not been established. The current study investigated the presence of ET-1 in human aqueous humor and evaluated changes in aqueous ET-1 levels in human DR patients compared with healthy controls. We also determined the relationship between ET-1 in the aqueous humor of patients with early non-proliferative DR (NPDR) with retinal hemodynamic alterations in early DR.

\section{Materials and methods}

\section{Participants}

All participants underwent a thorough ophthalmic examination to determine their eligibility for phacoemulsification. Other than the controls, the study included subjects with type 2 diabetes mellitus and no clinically visible or very mild NPDR without the presence of diabetic macular edema. The definition of mild NPDR was based on risk factors identified in the early treatment of diabetic retinopathy study for the development of macular edema (ETDRS ${ }^{35}$ ) and included patients with microaneurysms only, without macular edema. All subjects in the NPDR group were non-insulin dependent (NIDDM) and were using oral medications only to control their blood sugar levels. Subjects were excluded if they had any evidence of ocular diseases other than DR, a history of ocular surgery or laser procedures, and any history of systemic conditions other than diabetes and hypertension. Participants taking medications with known effects on blood flow (except for well-controlled systemic hypertension) were also excluded. None of the subjects smoked or had any respiratory diseases. This study was approved by the Research Ethics Board, University Health Network and by the Kensington Eye Institute, Toronto. Informed consent was obtained from each participant and the study was conducted according to the tenets of the Declaration of Helsinki.

\section{Sample size}

Using previously published data, total retinal blood flow (TRBF) was found to be significantly lower in patients with diabetes with NPDR compared to healthy controls (42.7 (SD \pm 7.5$)$ vs $33.0 \mu \mathrm{l} / \mathrm{min}(\mathrm{SD} \pm 9.2)$ ) (Tayyari et al $\left.{ }^{36}\right)$. The standardized effect size was calculated to be 1.054 and the resultant sample size, with an $\alpha$ of 0.05 and a $\beta$ of 0.8 (ie, power $=80 \%$ ), was 15 per group.

\section{Aqueous humor collection}

Informed consent was obtained from each participant. Pre-scheduled phacoemulsification was performed at the Kensington Eye Institute in Toronto, Canada. Before the intraocular surgery, undiluted samples of aqueous were obtained from all participants to measure ET-1. The method used in the collection of aqueous humor has been described in previous publications. ${ }^{37,38}$ The aqueous samples were thawed at room temperature, vortexed, and then spun at $13,000 \times \mathrm{g}$ for $5 \mathrm{~min}$ to remove any precipitates. Aqueous humor samples were then separated into aliquots of $30 \mu \mathrm{l}$. The samples were stored in an $-80^{\circ} \mathrm{C}$ freezer until analyses were performed.

\section{Measurement of aqueous ET-1 using multiplex bead analysis}

ET-1 concentrations were quantified using MILLIPLEX MAP technology, accurate for small sample volumes (Eve Technologies Corp, Calgary, AB, Canada), using the BioPlex 200 (Bio-Rad Laboratories, Inc., Hercules, CA, USA). Capture bead kits (R\&D, Minneapolis, MN, USA and Millipore, St. Charles, MO, USA) were used to determine ET-1 (Millipore). The limit of detection for ET-1 was $1.4 \mathrm{pg} / \mathrm{ml}$. The tests were performed in accordance with the manufacturer's instructions.

\section{Assessment of TRBF in participants}

Four weeks post-cataract surgery, participants were asked to return for non-invasive retinal blood flow measurements using the RTVue Doppler OCT (Optovue, Inc). Each study eye was dilated with $1 \%$ tropicamide and $2.5 \%$ phenylephrine eye drops. Briefly, this non-invasive system applies Fourier domain optical coherence tomography (FD-OCT) to extract and calculate volumetric blood flow. The FD-OCT system utilizes a double circular 
scan protocol (an inner and outer ring size of 3.40 and $3.75 \mu \mathrm{m}$, respectively) centered on the optic nerve head. Each ring transects each retinal arteriole and venule passing through the optic nerve head. The Doppler shift $(\Delta \mathrm{f})$ of the scattered light is the shift between two consecutive circular A-scans and is proportional to the flow velocity $(\mathrm{V})$, which is determined by the vector velocity of the moving erythrocytes. TRBF was calculated by summing the flow from all branch venules. Previous work has shown that retinal arterioles and venules have similar blood flow based on the assumption that inflow is equal to outflow in any sealed system. ${ }^{39}$ Further details about the instrument and principals on this system are described elsewhere. ${ }^{8,40-42}$

The analysis of images was semi-automatic and required manual refining of the vessel diameter which were identified using the computer algorithm. The raw Doppler OCT data are converted to Doppler OCT of retinal circulation grading software (version 2.1.1.4) for image grading and retinal blood flow calculation. For each image, each vessel is compared with a corresponding color fundus image and operators adjust to verify the type of vessel, vessel diameter boundaries, Doppler size, and intensity. The reproducibility of this semi-automatic method has been recently demonstrated by our group. ${ }^{41,43}$

Additionally, the TRBF is corrected for magnification error by axial length for each subject. In FD-OCT, the scan is actually based on a model eye with axial length of $24 \mathrm{~mm}$. Longer axial lengths decrease the magnification of the fundus image, reduce the transverse diameter of the vessel, and increase the apparent Doppler angle, making the velocity and flow appear artifactually low. As described by Srinivas et al, ${ }^{44}$ corrected TRBF can be obtained from the formula: corrected

$\mathrm{TRBF}=\mathrm{oTRBF} \times(\text { axial length/default axial length })^{2}$. Here oTRBF is the original total blood flow before eye length correction and the default axial length, which is a value of $24 \mathrm{~mm}$. The axial length-corrected TRBF was used in the statistical analyses.

\section{Statistical analysis}

All analyses were performed using Statistica System 11.0 software (SAS Institute Inc., Cary, NC, USA). Normality was assessed for all data and all results are presented as the mean \pm SD. Differences in TRBF and ET- 1 between control and NPDR groups were assessed using a Student's $t$-test on normally distributed data, and a Mann-Whitney $U$-test for nonparametric data. The correlation between TRBF, aqueous ET-1, and A1c was determined the Pearson Bivariate correlation test. Multiple linear regression analyses were performed to identify risk factors affecting TRBF and/or ET-1. The model for TRBF included independent variables; age,
Table 1 Demographics of the study population

\begin{tabular}{lccc}
\hline Variable & Control & NPDR & P-value \\
\hline Sample size & 15 & 15 & - \\
Age (years) & $68.0 \pm 6.32$ & $67.53 \pm 10.05$ & 0.883 \\
Duration of DM & - & $12.2 \pm 13.0$ & - \\
(years) & & & \\
Systolic BP (mmHg) & $129.29 \pm 17.78$ & $134.53 \pm 19.13$ & 0.451 \\
Diastolic BP (mmHg) & $76.71 \pm 9.56$ & $76.86 \pm 8.10$ & 0.963 \\
IOP (mmHg) & $14.85 \pm 1.74$ & $14.86 \pm 1.72$ & 0.988 \\
A1c (\%) & $5.53 \pm 0.23$ & $7.13 \pm 0.74$ & $<0.001^{*}$ \\
\hline
\end{tabular}

Abbreviations: A1c, glycosylated hemoglobin; BP, blood pressure; DM, diabetes mellitus; NPDR, non-proliferative diabetic retinopathy. *Indicates significant differences compared with controls $(P<0.05)$. Data are presented as mean \pm SD.

duration of diabetes mellitus, A1c, and ET-1. In all analyses, two-tailed $P<0.05$ was considered as statistical significance.

\section{Results}

\section{NPDR and control subject demographics}

We recruited a total of 30 participants and the characteristics are described in Table 1 . All subjects were age matched to controls $(P=0.88)$. The stage of retinopathy was confirmed by the collaborating ophthalmologist (SS). There were no significant differences in systolic blood pressure, diastolic blood pressure, or IOP between the NPDR and control groups $(P>0.451$ for all). A1c was significantly higher in the NPDR group compared with the controls $(P<0.001)$.

\section{Comparison of aqueous ET-1 in NPDR and control groups}

Aqueous ET-1 levels were analyzed using multiplex bead analysis. Aqueous ET-1 was significantly higher ( 35\%) in the NPDR group compared with the controls $(3.5 \pm 1.8$ vs $2.2 \pm 0.8 P=0.02$ ) (Figure 1a). Outliers were defined as values outside the $\pm 2 \mathrm{SD}$. Two control subjects were excluded from the study for this reason.

\section{Mean TRBF in NPDR and control groups}

TRBF was significantly lower $(\sim 28 \%)$ in the NPDR compared with the controls. The mean TRBF in the NPDR group was $34.5 \pm 9.1 \mu \mathrm{l} / \mathrm{min}$ and was $44.1 \pm 4.6 \mu \mathrm{l} / \mathrm{min}$ in the control group $(P=0.002)$, Figure $1 b$.

\section{Correlation between ET-1 and TRBF}

Pearson bivariate correlation test was performed to determine the association between aqueous ET-1 and 
a
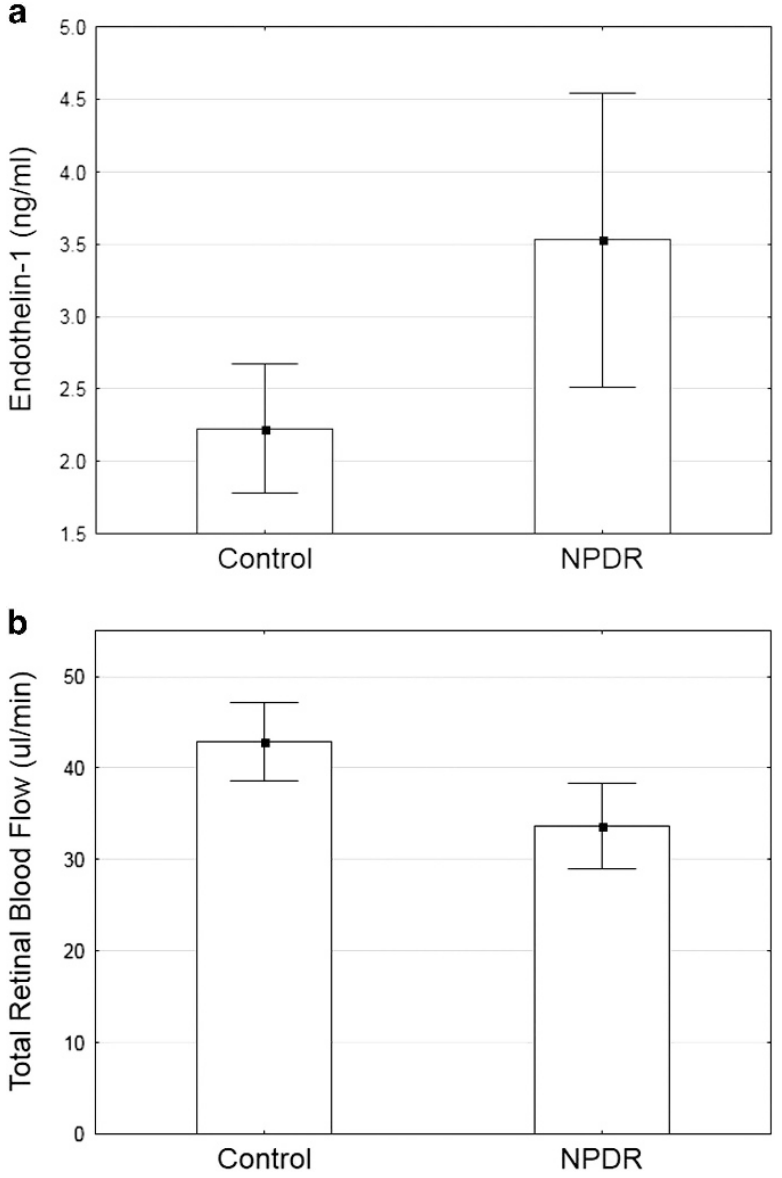

Figure 1 Plots of means (a) aqueous ET-1 as a function of group. ET-1 is significantly elevated in the NPDR group compared with the controls, $P=0.02$. (b) TRBF as a function of group. TRBF was significantly reduced in the NPDR group compared with the controls, $P=0.002$. Error bars indicate $95 \%$ confidence intervals of the mean. NPDR, non-proliferative diabetic retinopathy.

TRBF. There were no significant correlations found between aqueous ET- 1 and TRBF $(r=-0.11, P=0.63)$ across all groups. Additionally, there were no correlations found within the control group $(r=-0.26, P=0.45)$ or NPDR group $(r=0.15, P=0.63)$. There was a significant correlation found between A1c and ET-1 across all subjects $(r=0.69, P<0.01)$ as well as within the NDPR group ( $r=0.64, P=0.01$ ). Additionally, TRBF was found to be significantly correlated with A1c across all subjects $(r=-0.45, P=0.02)$. However, TRBF was not significantly associated with A1c within the NPDR group $(r=-0.13$, $P=0.65)$

\section{Multiple regression analyses}

Across controls and NPDR, multiple regression analyses showed no significant associations between TRBF and age, duration, A1c, or aqueous ET-1 $(P>0.07$ for all $)$ $\left(R^{2}=0.32\right.$, adjusted $\left.R^{2}=0.21, F(4,24)=2.8, P<0.04\right)$. Similar results were found in the NPDR group $(P>0.11$ for all) $\left(R^{2}=0.41\right.$, adjusted $R^{2}=0.18, F(4,10)=1.8$, $P<0.21)$.

\section{Discussion}

ET-1 plays an important role in retinal blood flow regulation and may be an important contributor to blood flow abnormalities in DR. ${ }^{34}$ The present study showed a significant elevation in aqueous ET-1 levels in patients with type 2 diabetes and NPDR compared with controls. Additionally, the NPDR group demonstrated significantly lower TRBF compared with the control group. Although there were no significant correlations between aqueous ET-1 and TRBF, the observed reduction in TRBF suggests that there is an initial vasoconstriction in type 2 diabetic patients with no or very mild NPDR, this effect could partially be attributed to diabetes-induced increase in ocular ET-1 levels.

This study is the first to report an increase $(\sim 35 \%)$ in aqueous ET-1 in early DR and normotensive diabetic patients compared with controls. Increased ET-1 has been found in the aqueous of patients with open-angle glaucoma where vascular dysfunction has a suspected pathogenic role. ${ }^{22}$ A positive correlation between plasma ET-1 levels, ${ }^{33}$ vitreous ET- $1,{ }^{30}$ and microangiopathy in patients with type 2 diabetes has been demonstrated. ET-1 is a potent vasoconstrictor involved in the pathogenesis of microangiopathy. ${ }^{44,45}$ Animal studies have shown that the direct injection of ET-1 into the vitreous of non-diabetic rats induces retinal vasoconstriction primarily via $\mathrm{ET}_{\mathrm{A}}$ receptors, which results in a decrease in retinal blood flow. ${ }^{4}$ Overall, there is evidence that increased ET-1 expression, together with $\mathrm{ET}_{\mathrm{A}}$ receptors located on smooth muscle cells of the retinal arterioles ${ }^{16}$ and pericytes, ${ }^{17}$ induces vascular constriction, resulting in a reduction in retinal blood flow during diabetes.

As mentioned above, vasoconstrictive factor ET-1 is known to have an important role in the regulation of retinal hemodynamics. Although aqueous ET-1 was upregulated, there was no significant correlation between aqueous humor level of ET-1 and TRBF $(P>0.634)$. In healthy individuals, ET-1 elicits a dosedependent vasoconstriction in retinal arteries and pericytes via $\mathrm{ET}_{\mathrm{A}}$ receptor interaction. ${ }^{19}$ However, studies have demonstrated a reduction in physiologic retinal blood flow in response to elevated ET-1 in the diabetic retina and this impaired response may be attributed to the alterations in $\mathrm{ET}_{\mathrm{A}}$ receptor expression. Additionally, during early stages of diabetes, retinal pericytes demonstrate prolonged desensitization to 
further ET-1 simulation after the first application of ET-1. Although the exact mechanism is still under investigation, hyperglycemia-induced alterations to $\mathrm{ET}_{\mathrm{A}}$ expression and/or pericyte desensitization may account for the absence of correlation between aqueous ET-1 and TRBF. Our results are consistent with previous studies by other investigators suggesting that a disturbance in ET-1 production contributes to the reduction in retinal blood flow observed in early diabetes.

The increased ET-1 expression has been associated with hyperglycemia-induced activation of diacylglycerol and protein kinase C (PKC) pathway during early diabetes. ${ }^{47}$ Studies have showed that activation of the PKC pathway has an important role in ET-1 expression and in the regulation of retinal hemodynamics. Diabetes-induced ET-1 upregulation associated with PKC activation has been shown to play an important role in the pathogenesis of DR. ${ }^{47-50}$ Consistently, the effect of diabetes on TRBF and ET-1 is highly correlated with hyperglycemia (A1c) $(P<0.01$ and $P=0.02$, respectively). This implies that ET-1 may be an early indicator of diabetes-induced changes in the ocular tissue.

Additionally, retinal microvascular abnormalities might be present in the capillary bed that we were not able to evaluate. Following 1 month of diabetes, animal models of diabetes demonstrated increased retinal ET-1 expression where increased resistance in the distal capillary bed was observed. ${ }^{51}$ Consistently, Polak et al ${ }^{52}$ have shown that intravitreal administration of exogenous ET-1 leads to reductions in retinal blood flow and ischemic-type properties in healthy participants. Additionally, recent in vivo models by Cheung et al ${ }^{21}$ demonstrate that overexpression of endogenous ET- 1 by endothelial cells induces mild transient inner retinal ischemia. Altogether, accumulating results suggest that diabetes-induced increases in ET-1 in the ocular tissue may be functionally important in early microcirculatory disruptions in DR. In addition, it is important to note that although ET-1 is a strong regulator of retinal blood flow, it is one of many factors that may directly or indirectly influence blood flow abnormalities in diabetes. It is possible that alteration in TRBF is not directly related to ET-1 alone but also involves the activation of other vasoactive factors, including nitric oxide (NO). Further studies are required to characterize the role of aqueous ET- 1 and other factors that may mediate retinal hemodynamic abnormalities in diabetes. Nonetheless, our results have extended these findings by showing disturbances in ocular ET-1 expression occur at the same time as retinal microcirculatory disruptions in early diabetes.

The importance of the mechanisms by which ET-1 modulates vasculopathy has been highlighted by non- ocular studies as well, where endothelial dysfunction plays a role. For instance, impaired endothelial macrovascular and microvascular reactivity to endogenous ET-1 has been demonstrated in patients with early glucose intolerance ${ }^{53}$ and non-insulin-dependent type II diabetes. ${ }^{54}$ Recently, Ahlborg et al ${ }^{55}$ showed that treatment using a dual ET antagonist in patients with insulin resistance improved insulin sensitivity, renal blood flow, and resistance, indicating an important role for endogenous ET-1 in endothelial dysfunction. Thus, the augmented ET-1 production and activity represents retinal vascular endothelial dysfunction, which is an early phenomenon in diabetes.

This study has several limitations. First, TRBF is the measurement of retinal venules draining into the optic nerve head, which may not reflect subtle localized changes in the capillary bed during diabetes. However, animal studies have shown that glucose-induced ET-1 expression alters retinal pericyte function, ${ }^{48,56}$ which can occur before alterations in retinal arteriole blood flow.

Additionally, blunted retinal blood flow responsiveness to exogenous ET-1 has been seen in humans with type 2 diabetes, ${ }^{57}$ suggesting changes in large vessels in terms of homeostatic blood flow might reflect changes in the capillary microvasculature. Second, we collected aqueous humor instead of vitreous samples even though the proximity of vitreous is closer to the retinal vasculature. However, enhanced synthesis of ET-1 expression by non-pigmented ciliary epithelial cells in the anterior chamber during hyperglycemia has been shown previously. ${ }^{57}$ Further, activation of PKC is an essential step for synthesis of ET-1 by the ciliary epithelia. ${ }^{58}$ Overall, there is reason to suggest that hyperglycemia in diabetes-mediated changes alters ET-1 expression in several ocular tissue, including the aqueous humor. Further studies are required to determine the mechanism of aqueous ET-1 and ET receptor interaction in mediating posterior segment physiology.

Although the exact pathogenic association between ET-1 and the development of retinal blood flow abnormalities remains to be clarified, it is noteworthy that aqueous humor cytokines like ET-1 are impacted in diabetes. This study demonstrated reduced retinal blood flow in patients with diabetes and no or very mild NPDR, which also corresponds with augmented ocular ET-1 levels. Together, measurement of aqueous humor ET-1 could potentially offer prognostic value in the management of DR. Further work is required to evaluate additional aqueous humor cytokines in the pathogenesis of early blood flow abnormalities in DR. 


\section{Summary}

\section{What was known before}

- Retinal blood flow alterations are an early indicator of DR.

- ET-1 plays an important role in retinal blood flow regulation and may be an important contributor to early blood flow abnormalities in DR.

- ET-1 in the aqueous may have an important physiological role in the regulation of the retinal circulation, especially in diabetes.

What this study adds

- Elevated aqueous ET-1 levels are consistent with reduced TRBF observed in early human DR.

- This work adds to our current understanding the physiological and pathophysiological implications of ET-1 in diabetes.

- Adds to the importance of aqueous humor cytokines as a prognostic indicator of retinal physiology.

\section{Conflict of interest}

$\mathrm{DH}$ and OT have significant financial interest in Optovue, a company that may have a commercial interest in the results of this research and technology. $\mathrm{CH}$ received support in terms of loaned equipment and funding for research. These potential conflicts of interest have been reviewed and managed by the Research Ethics Board of the University Health Network, Toronto and by Oregon Health \& Science University. The remaining authors declared no conflict of interest.

\section{Acknowledgements}

The authors thank Dr Ayda Shahidi from the University of Waterloo for research assistance, ophthalmology nursing team at the Toronto Western Hospital and Kensington Eye Institute for their surgical assistance. Supported by the Ontario Research Fund for Research Excellence (RE-04-034), NIH Grants DP3 DK104397, UL1TR000128, R01 EY023285, and by an anonymous donor.

\section{References}

1 Klein R, Klein BE. Are individuals with diabetes seeing better? A long-term epidemiological perspective. Diabetes 2010; 59: 1853-1860.

2 Yau JW, Rogers SL, Kawasaki R, Lamoureux EL, Kowalski JW, Bek T et al. Global prevalence and major risk factors of diabetic retinopathy. Diabetes Care 2012; 35: 556-564.

3 Bursell SE, Clermont AC, Oren B, King GL. The in vivo effect of endothelins on retinal circulation in nondiabetic and diabetic rats. Invest Ophthalmol Vis Sci 1995; 36: 596-607.
4 Takagi C, Bursell SE, Lin YW, Takagi H, Duh E, Jiang Z et al. Regulation of retinal hemodynamics in diabetic rats by increased expression and action of endothelin-1. Invest Ophthalmol Vis Sci 1996; 37: 2504-2518.

5 Feke GT, Buzney SM, Ogasawara H, Fujio N, Goger DG, Spack NP et al. Retinal circulatory abnormalities in type 1 diabetes. Invest Ophthalmol Vis Sci 1994; 35: 2968-2975.

6 Bursell SE, Clermont AC, Kinsley BT, Simonson DC, Aiello LM, Wolpert HA. Retinal blood flow changes in patients with insulin-dependent diabetes mellitus and no diabetic retinopathy. Invest Ophthalmol Vis Sci 1996; 37: 886-897.

7 Nagaoka T, Sato E, Takahashi A, Yokota H, Sogawa K, Yoshida A. Impaired retinal circulation in patients with type 2 diabetes mellitus: retinal laser Doppler velocimetry study. Invest Ophthalmol Vis Sci 2010; 51: 6729-6734.

8 Wang Y, Fawzi A, Tan O, Gil-Flamer J, Huang D. Retinal blood flow detection in diabetic patients by Doppler Fourier domain optical coherence tomography. Opt Express 2009; 17: 4061-4073.

9 Yao K, Tschudi M, Flammer J, Luscher TF. Endotheliumdependent regulation of vascular tone of the porcine ophthalmic artery. Invest Ophthalmol Vis Sci 1991; 32: 1791-1798.

10 Haefliger IO, Flammer J, Beny JL, Luscher TF. Endotheliumdependent vasoactive modulation in the ophthalmic circulation. Prog Retin Eye Res 2001; 20: 209-225.

11 Furchgott RF, Vanhoutte PM. Endothelium-derived relaxing and contracting factors. FASEB J 1989; 3: 2007-2018.

12 Narayan S, Prasanna G, Krishnamoorthy RR, Zhang X, Yorio T. Endothelin-1 synthesis and secretion in human retinal pigment epithelial cells (ARPE-19): differential regulation by cholinergics and TNF-alpha. Invest Ophthalmol Vis Sci 2003; 44: 4885-4894.

13 Lepple-Wienhues A, Becker M, Stahl F, Berweck S, Hensen J, Noske W et al. Endothelin-like immunoreactivity in the aqueous humour and in conditioned medium from cultured ciliary epithelial cells. Curr Eye Res 1992; 11: 1041-1046.

14 MacCumber MW, Jampel HD, Snyder SH. Ocular effects of the endothelins. Abundant peptides in the eye. Arch Ophthalmol 1991; 109: 705-709.

15 Chakravarthy U, Douglas AJ, Bailie JR, McKibben B, Archer DB. Immunoreactive endothelin distribution in ocular tissues. Invest Ophthalmol Vis Sci 1994; 35: 2448-2454.

16 Hein TW, Ren Y, Yuan Z, Xu W, Somvanshi S, Nagaoka T et al. Functional and molecular characterization of the endothelin system in retinal arterioles. Invest Ophthalmol Vis Sci 2009; 50: 3329-3336.

17 Chakravarthy U, Gardiner TA, Anderson P, Archer DB, Trimble ER. The effect of endothelin 1 on the retinal microvascular pericyte. Microvasc Res 1992; 43: 241-254.

18 Ramachandran E, Frank RN, Kennedy A. Effects of endothelin on cultured bovine retinal microvascular pericytes. Invest Ophthalmol Vis Sci 1993; 34: 586-595.

19 Polak K, Petternel V, Luksch A, Krohn J, Findl O, Polska E et al. Effect of endothelin and BQ123 on ocular blood flow parameters in healthy subjects. Invest Ophthalmol Vis Sci 2001; 42: 2949-2956. 
20 Trick GL, Berkowitz BA. Retinal oxygenation response and retinopathy. Prog Retin Eye Res 2005; 24: 259-274.

21 Cheung SS, Leung JW, Lam AK, Lam KS, Chung SS, Lo AC et al. Selective over-expression of endothelin-1 in endothelial cells exacerbates inner retinal edema and neuronal death in ischemic retina. PLoS One 2011; 6: e26184.

22 Sin BH, Song BJ, Park SP. Aqueous vascular endothelial growth factor and endothelin-1 levels in branch retinal vein occlusion associated with normal tension glaucoma. J Glaucoma 2013; 22: 104-109.

23 Patel C, Narayanan SP, Zhang W, Xu Z, Sukumari-Ramesh S, Dhandapani KM et al. Activation of the endothelin system mediates pathological angiogenesis during ischemic retinopathy. Am J Pathol 2014; 184: 3040-3051.

24 Noske W, Hensen J, Wiederholt M. Endothelin-like immunoreactivity in aqueous humor of patients with primary open-angle glaucoma and cataract. Graefes Arch Clin Exp Ophthalmol 1997; 235: 551-552.

25 Tezel G, Kass MA, Kolker AE, Becker B, Wax MB. Plasma and aqueous humor endothelin levels in primary open-angle glaucoma. J Glaucoma 1997; 6: 83-89.

26 Cellini M, Strobbe E, Gizzi C, Balducci N, Toschi PG, Campos EC. Endothelin-1 plasma levels and vascular endothelial dysfunction in primary open angle glaucoma. Life Sci 2012; 91: 699-702.

27 Chakravarthy U, Hayes RG, Stitt AW, Douglas A. Endothelin expression in ocular tissues of diabetic and insulin-treated rats. Invest Ophthalmol Vis Sci 1997; 38: 2144-2151.

28 McGinty A, Scholfield CN, Liu WH, Anderson P, Hoey DE, Trimble ER. Effect of glucose on endothelin-1-induced calcium transients in cultured bovine retinal pericytes. J Biol Chem 1999; 274: 25250-25253.

29 Oku H, Kida T, Sugiyama T, Hamada J, Sato B, Ikeda T. Possible involvement of endothelin- 1 and nitric oxide in the pathogenesis of proliferative diabetic retinopathy. Retina 2001; 21: 647-651.

30 Patel JI, Saleh GM, Hykin PG, Gregor ZJ, Cree IA. Concentration of haemodynamic and inflammatory related cytokines in diabetic retinopathy. Eye 2008; 22: 223-228.

31 Roldan-Pallares M, Rollin R, Martinez-Montero JC, Fernandez-Cruz A, Bravo-Llata C, Fernandez-Durango R. Immunoreactive endothelin-1 in the vitreous humor and epiretinal membranes of patients with proliferative diabetic retinopathy. Retina 2007; 27: 222-235.

32 Adamiec-Mroczek J, Oficjalska-Mlynczak J, Misiuk-Hojlo M. Roles of endothelin-1 and selected proinflammatory cytokines in the pathogenesis of proliferative diabetic retinopathy: analysis of vitreous samples. Cytokine 2010; 49: 269-274.

33 Takahashi K, Ghatei MA, Lam HC, O'Halloran DJ, Bloom SR. Elevated plasma endothelin in patients with diabetes mellitus. Diabetologia 1990; 33: 306-310.

34 Kawamura M, Ohgawara H, Naruse M, Suzuki N, Iwasaki N, Naruse $\mathrm{K}$ et al. Increased plasma endothelin in NIDDM patients with retinopathy. Diabetes Care 1992; 15: 1396-1397.

35 Early Treatment Diabetic Retinopathy Study Research Group. (ETDRS) Fundus photographic risk factors for progression of diabetic retinopathy (ETDRS report no. 12). Ophthalmology 1991; 98: 823-833.

36 Tayyari F, Khuu LA, Flanagan JA, Singer S, Brent $\mathrm{MH}$, Hudson C. Retinal blood flow and retinal blood oxygen saturation in mild-to-moderate diabetic retinopathy. Invest Ophthalmol Vis Sci 2015; 56: 6796-6800.

37 Bennett KL, Funk M, Tschernutter M, Breitwieser FP, Planyavsky M, Ubaida Mohien C et al. Proteomic analysis of human cataract aqueous humour: comparison of onedimensional gel LCMS with two-dimensional LCMS of unlabelled and iTRAQ(R)-labelled specimens. J Proteomics 2011; 74: 151-166.

38 Dong N, Xu B, Wang B, Chu L. Study of 27 aqueous humor cytokines in patients with type 2 diabetes with or without retinopathy. Mol Vis 2013; 19: 1734-1746.

39 Feke GT, Tagawa H, Deupree DM, Goger DG, Sebag J, Weiter JJ. Blood flow in the normal human retina. Invest Ophthalmol Vis Sci 1989; 30: 58-65.

40 Wang Y, Lu A, Gil-Flamer J, Tan O, Izatt JA, Huang D. Measurement of total blood flow in the normal human retina using Doppler Fourier-domain optical coherence tomography. Br J Ophthalmol 2009; 93: 634-637.

41 Tayyari F, Yusof F, Vymyslicky M, Tan O, Huang D, Flanagan JG et al. Variability and repeatability of quantitative, fourier-domain optical coherence tomography Doppler blood flow in young and elderly healthy subjects. Invest Ophthalmol Vis Sci 2014; 55: 7716-7725.

42 Shahidi AM, Patel SR, Huang D, Tan O, Flanagan JG, Hudson C. Assessment of total retinal blood flow using Doppler Fourier domain optical coherence tomography during systemic hypercapnia and hypocapnia. Physiol Rep 2014; 2: e12046.

43 Konduru RK, Tan O, Nittala MG, Huang D, Sadda SR. Reproducibility of retinal blood flow measurements derived from semi-automated Doppler OCT analysis. Ophthalmic Surg Lasers Imaging 2012; 43: 25-31.

44 Srinivas S, Tan O, Wu S, Nittala MG, Huang D, Varma R et al. Measurement of retinal blood flow in normal chineseamerican subjects by Doppler fourier-domain optical coherence tomography. Invest Ophthalmol Vis Sci 2015; 56: 1569-1574.

45 Bohm F, Pernow J. The importance of endothelin- 1 for vascular dysfunction in cardiovascular disease. Cardiovasc Res 2007; 76: 8-18.

46 Kitada K, Ohkita M, Matsumura Y. Pathological importance of the endothelin-1/ET(B) receptor system on vascular diseases. Cardiol Res Pract 2012; 2012: 731970.

47 Wiwanitkit V. Endothelin-1 and protein kinase C co-expression in the pathogenesis of diabetic retinopathy. J Diabetes Complications 2007; 21: 359-362.

48 Park JY, Takahara N, Gabriele A, Chou E, Naruse K, Suzuma K et al. Induction of endothelin-1 expression by glucose: an effect of protein kinase $\mathrm{C}$ activation. Diabetes 2000; 49: 1239-1248.

49 Way KJ, Katai N, King GL. Protein kinase C and the development of diabetic vascular complications. Diabet Med 2001; 18: 945-959.

50 Aiello LP. The potential role of PKC $\beta$ in diabetic retinopathy and macular edema. Surv Ophthalmol 2002; 47: S263-S269.

51 Deng D, Evans T, Mukherjee K, Downey D, Chakrabarti S. Diabetes-induced vascular dysfunction in the retina: role of endothelins. Diabetologia 1999; 42: 1228-1234.

52 Polak K, Luksch A, Frank B, Jandrasits K, Polska E, Schmetterer L. Regulation of human retinal blood flow by endothelin-1. Exp Eye Res 2003; 76: 633-640.

53 Diehl KJ, Templeton DL, Ma J, Weil BR, Greiner JJ, Stauffer BL et al. Impaired fasting blood glucose is associated 
with increased endothelin-1 vasoconstrictor tone.

Atherosclerosis 2013; 229: 130-133.

54 McAuley DF, Nugent AG, McGurk C, Maguire S, Hayes JR, Johnston GD. Vasoconstriction to endogenous endothelin-1 is impaired in patients with type II diabetes mellitus. Clin Sci (Lond) 2000; 99: 175-179.

55 Ahlborg G, Shemyakin A, Bohm F, Gonon A, Pernow J. Dual endothelin receptor blockade acutely improves insulin sensitivity in obese patients with insulin resistance and coronary artery disease. Diabetes Care 2007; 30: 591-596.
56 Kawamura H, Oku H, Li Q, Sakagami K, Puro DG. Endothelin-induced changes in the physiology of retinal pericytes. Invest Ophthalmol Vis Sci 2002; 43: 882-888.

57 Wang Z, Yadav AS, Leskova W, Harris NR. Attenuation of streptozotocin-induced microvascular changes in the mouse retina with the endothelin receptor $\mathrm{A}$ antagonist atrasentan. Exp Eye Res 2010; 91: 670-675.

58 Prasanna G, Dibas A, Tao W, White K, Yorio T. Regulation of endothelin-1 in human non-pigmented ciliary epithelial cells by tumor necrosis factor-alpha. Exp Eye Res 1998; 66: 9-18. 